UW Biostatistics Working Paper Series

$5-25-2008$

\title{
Semiparametric methods for evaluating the covariate-specific predictiveness of continuous markers in matched case-control studies
}

Ying Huang

Fred Hutchinson Cancer Research Center, ying@u.washington.edu

Margaret S. Pepe

University of Washington, mspepe@u.washington.edu

\section{Suggested Citation}

Huang, Ying and Pepe, Margaret S., "Semiparametric methods for evaluating the covariate-specific predictiveness of continuous markers in matched case-control studies" (May 2008). UW Biostatistics Working Paper Series. Working Paper 329.

http://biostats.bepress.com/uwbiostat/paper329

This working paper is hosted by The Berkeley Electronic Press (bepress) and may not be commercially reproduced without the permission of the copyright holder.

Copyright $(\mathcal{C} 2011$ by the authors 


\section{Introduction}

New technologies, such as gene expression microarrays and protein mass spectrometry, promise a multitude of biomarkers for detecting disease and predicting future events. Statistical methodology is needed to critically evaluate them. Biomarkers differ in their purposes and consequently demand different criteria for evaluation. For example, diagnostic markers are used to classify people as diseased or nondiseased. Their performance is typically evaluated with measures like sensitivity, specificity, and the ROC curve. Risk prediction markers, on the other hand, are utilized to predict the risk of having or getting a disease or other event. For risk prediction markers, measures that quantify their abilities to stratify risk are most relevant (Cook, 2007). Pepe et al. (2007) and Huang et al. (2007) proposed a graphical tool, the predictiveness curve to characterize the population distribution of predicted risk. Let $D$ be a binary outcome, and $Y$ be the markers of interest, and let $\operatorname{Risk}(y)=P(D=1 \mid Y=y)$ be the risk predicted for subjects with marker value $Y=y$. We write the predictiveness curve $R(v)$ vs $v$, where $R(v)$ is the $100 \times v^{t h}$ percentile of $\operatorname{Risk}(Y)$. Equivalently we plot $p$ vs $F_{R}(p)$, where $F_{R}(p)$ is the cumulative distribution of risk in the population, $R^{-1}(p)=F_{R}(p)$.

The distribution of $\operatorname{Risk}(Y)$ can be used to compare risk prediction markers or models. It provides a common scale for comparing models. A better model for risk stratification will have larger variability in risk percentiles. Equivalently, it will assign more subjects into low and high risk ranges.

As pointed out by Huang et al. (2007), the predictiveness of a marker depends on the target population. If there are covariates that define subpopulations, then a marker's covariate-specific predictiveness should be explored in order to identify subpopulations where it is useful for risk stratification and subpopulations where it is not. Another motivation for considering covariate-specific curves is for individual decision making about having a marker measured or not. A subject may decide that it is valuable to ascertain the value of a marker for him/her only if there is a reasonably large chance that his/her risk calculated on the basis of marker and covariates will differ substantially from that calculated only on the basis of covariates. Covariate-specific predictiveness curves also address this question as we will illustrate.

There are two ways in which covariates can impact a marker's predictiveness. Covariates may be predictors themselves, possibly interacting with the marker's effect on risk. In other words, covariates can affect the shape and height of the risk as a function of $Y$. On the other hand, the distribution of the marker may 
vary with covariates. Both effects impact on the distribution of risk, i.e. the predictiveness curve.

Let $Z$ be covariates of interest, let $\operatorname{Risk}(Y, Z)=P(D=1 \mid Y, Z)$, then the covariate-specific predictiveness curve for $Y$ given $Z=z$ is the curve $R_{z}(v)$ vs $v$, where $R_{z}(v)$ is the $100 \times v^{t h}$ percentile of $\operatorname{Risk}(Y, Z)$ in the population with $Z=z$. Estimation of the covariate-specific predictiveness curve with data from a cohort design has been studied in Huang et al. (2007). Since case-control studies are more frequently conducted, particularly in the early phases of biomarker development (Pepe et al., 2001), it is important to develop estimators for this type of design as well. Here we consider case-control study designs. Moreover, our methods accommodate matching where controls are frequency matched to cases in regards to a subset of covariates.

To fix ideas consider the following example of a study to evaluate serum creatinine as a predictor of risk of renal artery stenosis in patients with therapy resistant hypertension. A large cohort of subjects undergoing renal arteriography constitutes the patient cohort for a case-control study of serum creatinine. Details are provided in Section 4. Baseline risk of significant stenosis is calculated on the basis of several covariates including age, gender, smoking, etc. For illustration, we consider risk values above 0.4 as high enough to routinely recommend renal arteriography and risk values below 0.1 as low enough to discourage the practice. Figure 1 shows the predictiveness curves for serum creatine in subjects with baseline risk categorized as low, medium, and high. We see that among subjects originally deemed medium risk according to baseline factors, $24.8 \%$ are reclassified as low risk and $7.1 \%$ reclassified as high risk after including serum creatinine in the risk model. On the other hand, only a small fraction of subjects in the high or low risk categories according to baseline factors move to the medium risk designation after serum creatinine is included, and none move from the high risk to low risk category or vice versa. Therefore, on average ascertainment of serum creatinine seems to be most useful for subjects with medium baseline risk since in this group a substantial proportion are moved across risk thresholds that affect medical decisions. Cook (2007) proposed a simplified version of this sort of approach to evaluating the incremental value of C-reactive protein for cardiovascular risk assessment.

While predictiveness curves specific to baseline risk categories provide a big picture of the marker's incremental value in terms of affecting medical decisions in subpopulations, another kind of predictiveness curve which is specific to an individual's baseline covariate values is more useful for individual decision 
making. In Figure 2 the covariate-specific curves are for individuals with specific values for the baseline covariates. For example, subject 2 has baseline risk equal to $38.1 \%$. His predictiveness curve indicates that if serum creatinine is obtained from him, he has $0.5 \%$ chance of being classified as low risk. If his personal threshold for risk is low and he will opt for renal arteriography unless he is deemed to have risk $<10 \%$, there is no point in ascertaining serum creatine for him, because almost certainly his risk calculated with serum creatinine in addition to baseline covariates will exceed $10 \%$.

In this paper we developed methodology to estimate and make inference about covariate-specific predictiveness curves, exemplified in Figures 1 and 2.

\section{Method}

We consider a case-control design where cases and controls are frequency matched within $\mathcal{S}$ different strata. The special case where there is no matching corresponds to $\mathcal{S}=1$. Let $S$ be the indicator for the matching stratum, taking values from 1 to $\mathcal{S}$. We are interested in estimating the predictiveness curve for marker $Y$ given values of covariate $Z$, assuming $\rho_{s}=P(D=1 \mid S=s)$, the disease prevalence within stratum $s$ is apriori known. Consider the scenario where matching stratum $S$ can be written as a function of $Z, S(Z)$. An implication of this functional relationship between $S$ and $Z$ is that risk of disease conditional on marker $Y$ and covariate $Z$ is independent of stratum $S$. That is, $P(D=1 \mid Y, Z, S)=P(D=1 \mid Y, Z)$, which we denote by $G(\theta, Y, Z)$, where $\theta$ is the risk model parameter. This is satisfied for example if strata are defined by a subset of covariates. Refer to the example in Section 1. Suppose samples are matched on baseline risk category, we have $s=1,2,3$. Marker $Y$ is serum creatinine. Covariate $Z$ is baseline risk category in Figure 1 and baseline covariates in Figure 2 .

Consider a logistic model where risk conditional on covariate value is monotone increasing as marker value increases

$$
\operatorname{logit}\{P(D=1 \mid Y, Z)\}=\operatorname{logit}\{G(\theta, Y, Z)\}=\theta_{0}+\kappa\left(\theta_{1}, Y, Z\right)
$$

where $\theta=\left(\theta_{0}, \theta_{1}\right)^{T}$. Here $\kappa\left(\theta_{1}, Y, Z\right)$ is a pre-defined monotone increasing function for $Y$ given $Z=z$. For

example, $\kappa\left(\theta_{1}, Y, Z\right)$ might be $\theta_{11} Y+\theta_{12}^{T} Z$, the linear logistic model, or more flexibly $\theta_{11} Y^{\left(\theta_{13}\right)}+\theta_{12}^{T} Z$, where $Y^{\left(\theta_{13}\right)}$ is the Box-Cox transformation (Cole and Green, 1992). Moreover, interaction between $Y$ and $Z$ might also be included. Note that under the monotone increasing risk assumption, the curve $R_{z}(v)$ vs $v$ can be 
written as $P(D=1 \mid Y, z)$ vs $F_{z}^{-1}(Y)$ where $F_{z}(y)=P(Y \leq y \mid Z=z)$ is the CDF of $Y$ when $Z=z$. This suggests estimation of the curve can be achieved in two steps: estimation of the risk model and estimation of the covariate-specific marker distribution. We take advantage of this in our proposed methods.

\subsection{Discrete Covariate}

Suppose the covariate of interest is discrete with $\mathcal{Z}$ categories. Let $Z$ be the covariate group indicator, taking values from 1 to $\mathcal{Z}$. For example, in a study where cases and controls are frequency matched by age subgroup, covariates of interest might be the combination of age subgroup and gender. A default strategy of estimating the predictiveness curve for marker $Y$ within a particular covariate group is to use sample from that covariate group only. However, when risk model is not fully saturated (i.e. including main effects for all covariate categories and their interaction with the marker), borrowing information across covariate groups is more efficient.

Consider the risk model

$$
\operatorname{logit}\{P(D=1 \mid Y, Z, S)\}=\operatorname{logit}\{G(\theta, Y, Z)\}=\theta_{0}+\theta_{1} Y+\theta_{2}^{T} Z^{M}+\theta_{3}^{T} Y Z^{I},
$$

where $\theta=\left(\theta_{0}, \theta_{1}, \theta_{2}, \theta_{3}\right)^{T}$. Here $Z^{M}, Z^{I}$ are vectors indexing subsets of covariate group. For example, suppose covariate $Z$ is the combination of age subgroup and gender, we can include $Z^{M}$ to index main effect for age subgroup and main effect for gender, and include $Z^{I}$ to index interaction between marker and gender only. The interaction terms vanishes when $Z^{I}$ is of length 0 . Let $\mathcal{L} \mathrm{R}_{z}$ be the likelihood ratio of $Y$ conditional on covariate value $z$, which belongs to matching stratum $S(z)$, we have

$$
\begin{aligned}
\mathcal{L R}_{z}(y) & =\frac{P(Y=y \mid D=1, Z=z)}{P(Y=y \mid D=0, Z=z)} \\
& =\frac{P(D=1 \mid Y=y, Z=z)}{P(D=0 \mid Y=y, Z=z)} \frac{P(D=0 \mid Z=z)}{P(D=1 \mid Z=z)} \\
& =\exp \left\{\theta_{0}+\eta(z)+\theta_{1} y+\theta_{2}^{T} z^{M}+\theta_{3}^{T} y z^{I}\right\},
\end{aligned}
$$

where $\eta(z)=\log \{P(D=0 \mid Z=z) / P(D=1 \mid Z=z)\}$.

When the matching stratum $S$ is the same as the covariate group, $P(D=1 \mid Z=z)$ is known by assumption, and so is $\eta(z)$. Consider now settings where the matching stratum is a proper subset of the covariate group. If information about $P(D=1 \mid Z=z)$ is available, it can be directly utilized. However, if disease prevalence within a covariate group is not available, $P(D=1 \mid Z=z)$ and $\eta(z)$ can be estimated 
from the case-control sample because by Bayes' theorem, for $s=S(z)$,

$$
\begin{aligned}
\eta(z) & =\log \left\{\frac{P(D=0 \mid Z=z, S=s)}{P(D=1 \mid Z=z, S=s)}\right\} \\
& =\log \left\{\frac{P(D=0 \mid S=s)}{P(D=1 \mid S=s)}\right\}+\log \left\{\frac{P(Z=z \mid D=0, S=s)}{P(Z=z \mid D=1, S=s)}\right\} \\
& =\log \left(\frac{1-\rho_{s}}{\rho_{s}}\right)+\log \left\{\frac{P(Z=z \mid D=0, S=s)}{P(Z=z \mid D=1, S=s)}\right\}
\end{aligned}
$$

the first term in (3) is known, and for the second term, we can estimate $P(Z=z \mid D=0, S=s)$ and $P(Z=z \mid D=1, S=s)$ empirically from the case-control sample. Henceforth we use $\eta(z)$ to represent the value that is known or estimated.

Suppose $n_{D}$ cases and $n_{\bar{D}}$ controls are sampled in the study. Let $U_{s}$ be the set of subjects within matching stratum $s$ with $U_{D s}$ and $U_{\bar{D} s}$ being the subsets of cases and controls respectively. Let $i$ index subject. We maximize the empirical likelihood (Owen, 1988, 1990; Qin and Lawless, 1994) of observing the marker and the covariates values in the matched case-control sample.

$$
\begin{aligned}
\mathcal{L} & =\prod_{s=1}^{\mathcal{S}}\left\{\prod_{i \in U_{\bar{D} s}} P\left(Y_{i}, Z_{i} \mid D_{i}\right) \prod_{i \in U_{D s}} P\left(Y_{i}, Z_{i} \mid D_{i}\right)\right\} \\
& =\prod_{s=1}^{\mathcal{S}}\left\{\prod_{i \in U_{\bar{D} s}} P\left(Y_{i} \mid Z_{i}, D_{i}\right) P\left(Z_{i} \mid D_{i}\right) \prod_{i \in U_{D s}} P\left(Y_{i} \mid Z_{i}, D_{i}\right) P\left(Z_{i} \mid D_{i}\right)\right\} \\
& \propto \prod_{s=1}^{\mathcal{S}}\left\{\prod_{i \in U_{\bar{D} s}} P\left(Y_{i} \mid Z_{i}, D_{i}\right) \prod_{i \in U_{D s}} P\left(Y_{i} \mid Z_{i}, D_{i}\right)\right\}
\end{aligned}
$$

Define $F_{z}, F_{D z}$, and $F_{\bar{D} z}$ to be the cumulative distribution functions for marker $Y$ in covariate group $z$ in the general, diseased, and non-diseased populations. With a slight abuse of notation, let $U_{z}$ be the set of subjects within covariate group $z$ with $U_{D z}$ and $U_{\bar{D} z}$ being the subsets of cases and controls respectively, and let $n_{z}, n_{D z}$, and $n_{\bar{D} z}$ be the corresponding sample sizes. Denote by $p_{i z}$ the density of $Y$ for the $i^{t h}$ subject 
in covariate group $z$ in the non-diseased population. Note that (4) can be rewritten as

$$
\begin{aligned}
\mathcal{L}\left(\theta, F_{\bar{D} z}\right) & =\prod_{z=1}^{\mathcal{Z}}\left\{\prod_{i \in U_{\bar{D} z}} d F_{\bar{D} z}\left(Y_{i}\right) \prod_{i \in U_{D z}} d F_{D z}\left(Y_{i}\right)\right\} \\
& =\prod_{z=1}^{\mathcal{Z}}\left\{\prod_{i \in U_{\bar{D} z}} d F_{\bar{D} z}\left(Y_{i}\right) \prod_{i \in U_{D z}} \mathcal{L} \mathrm{R}_{z}\left(Y_{i}\right) d F_{\bar{D} z}\left(Y_{i}\right)\right\} \\
& =\prod_{z=1}^{\mathcal{Z}}\left\{\prod_{i \in U_{z}} d F_{\bar{D} z}\left(Y_{i}\right) \prod_{i \in U_{D z}} \mathcal{L} \mathrm{R}_{z}\left(Y_{i}\right)\right\} \\
& =\prod_{z=1}^{\mathcal{Z}}\left[\prod_{i \in U_{z}} p_{i z} \prod_{i \in U_{D z}} \exp \left\{\theta_{0}+\eta(z)+\theta_{1} Y_{i}+\theta_{2}^{T} Z_{i}^{M}+\theta_{3}^{T} Y_{i} Z_{i}^{I}\right\}\right]
\end{aligned}
$$

subject to the restrictions $p_{i z}>0, \sum_{i \in U_{z}} p_{i k}=1$, and $\sum_{i \in U_{z}} p_{i z} \mathcal{L} \mathrm{R}_{z}\left(Y_{i}\right)=1$, for $z=1, \ldots$, $\mathcal{Z}$. It is equivalent to maximize

$$
\begin{aligned}
l & =\sum_{z=1}^{\mathcal{Z}} \sum_{i \in U_{z}} \log \left(p_{i z}\right)+\sum_{z=1}^{\mathcal{Z}} \sum_{i \in U_{D z}}\left\{\theta_{0}+\eta(z)+\theta_{1} Y_{i}+\theta_{2}^{T} Z_{i}^{M}+\theta_{3}^{T} Y_{i} Z_{i}^{I}\right\} \\
& +\sum_{z=1}^{\mathcal{Z}} \lambda_{1 z}\left(1-\sum_{i \in U_{z}} p_{i z}\right) \\
& +\sum_{z=1}^{\mathcal{Z}} \lambda_{2 z}\left[\sum_{i \in U_{z}} p_{i z}-\sum_{i \in U_{z}} p_{i z} \exp \left\{\theta_{0}+\eta(z)+\theta_{1} Y_{i}+\theta_{2}^{T} Z_{i}^{M}+\theta_{3}^{T} Y_{i} Z_{i}^{I}\right\}\right],
\end{aligned}
$$

where $\lambda_{1 z}, \lambda_{2 z}$ are Lagrange multipliers.

Setting the first derivatives with respect to $p_{i z}, \theta_{2}$ and $\theta_{0}$ to be zero, we get

$$
\begin{aligned}
\frac{\partial l}{\partial p_{i z}} & =\frac{1}{p_{i z}}-\lambda_{1 z}-\lambda_{2 z}\left[\exp \left\{\theta_{0}+\eta(z)+\theta_{1} Y_{i}+\theta_{2}^{T} Z_{i}^{M}+\theta_{3}^{T} Y_{i} Z_{i}^{I}\right\}-1\right]=0 \\
& \Rightarrow p_{i z}=\frac{1}{\lambda_{1 z}+\lambda_{2 z}\left[\exp \left\{\theta_{0}+\eta(z)+\theta_{1} Y_{i}+\theta_{2}^{T} Z_{i}^{M}+\theta_{3}^{T} Y_{i} Z_{i}^{I}\right\}-1\right]} \\
\sum_{i \in U_{z}} \frac{\partial l}{\partial p_{i z}} p_{i z} & =n_{k}-\lambda_{1 z}-\lambda_{2 z}+\lambda_{2 z}=0 \\
& \Rightarrow \lambda_{1 z}=n_{z} \\
\frac{\partial l}{\partial \theta_{0}} & =n_{D}-\sum_{z=1}^{Z} \lambda_{2 z} \sum_{i \in U_{z}} p_{i z} \exp \left\{\theta_{0}+\eta(z)+\theta_{1} Y_{i}+\theta_{2}^{T} Z_{i}^{M}+\theta_{3}^{T} Y_{i} Z_{i}^{I}\right\}=0 \\
& \Rightarrow \sum_{z=1}^{\mathcal{Z}} \lambda_{2 z}=n_{D} .
\end{aligned}
$$

Methods for solving $\lambda_{2 z}$ and $\theta$ are derived in the appendix. In the special case where $Z^{M}$ is the covariate group indicator, a closed-form solution does exist and the remainder of this section focuses on that special case. That is, suppose $Z^{M}$ is a length $\mathcal{Z}-1$ vector of dummy variables indicating covariate group, with the 
$i^{\text {th }}$ element being $I(Z=i+1), i=1, \ldots, \mathcal{Z}-1$, we have

$$
\begin{aligned}
\frac{\partial l}{\partial \theta_{2}} & =\sum_{z=1}^{\mathcal{Z}} \sum_{i \in U_{D z}} Z_{i}-\sum_{z=1}^{\mathcal{Z}} \lambda_{2 k} \sum_{i \in U_{z}} Z_{i}^{M} p_{i k} \exp \left\{\theta_{0}+\eta(z)+\theta_{1} Y_{i}+\theta_{2}^{T} Z_{i}^{M}+\theta_{3}^{T} Y_{i} Z_{i}^{I}\right\}=0 \\
& \Rightarrow\left[\lambda_{2 z}\right]_{z=2, \ldots, \mathcal{Z}}=\sum_{z=1}^{\mathcal{Z}} \sum_{i \in U_{D z}} Z_{i}^{M}=\left[n_{D z}\right]_{z=2, \ldots, \mathcal{Z}} .
\end{aligned}
$$

Thus $\lambda_{2 z}=n_{D z}, z=1, \ldots, \mathcal{Z}$. We have

$$
p_{i z}=\frac{1}{n_{\bar{D} z}+n_{D z} \mathcal{L} \mathrm{R}_{z}\left(Y_{i}\right)}=\frac{1}{n_{\bar{D} z}+n_{D z} \exp \left\{\theta_{0}+\eta(z)+\theta_{1} Y_{i}+\theta_{2}{ }^{T} Z_{i}^{M}+\theta_{3}{ }^{T} Y_{i} Z_{i}^{I}\right\}} .
$$

Substituting $p_{i z}$ into (5) we have

$$
\begin{aligned}
l & =\sum_{z=1}^{\mathcal{Z}} \sum_{i \in U_{z}}-\log \left[n_{\bar{D} z}+n_{D z} \exp \left\{\theta_{0}+\eta(z)+\theta_{1} Y_{i}+\theta_{2}^{T} Z_{i}^{M}+\theta_{3}^{T} Y_{i} Z_{i}^{I}\right\}\right] \\
& +\sum_{z=1}^{\mathcal{Z}} \sum_{i \in U_{D z}}\left\{\theta_{0}+\eta(z)+\theta_{1} Y_{i}+\theta_{2}^{T} Z_{i}^{M}+\theta_{3}^{T} Y_{i} Z_{i}^{I}\right\} .
\end{aligned}
$$

Thus the maximum likelihood estimators $\hat{\theta}_{0}, \hat{\theta}_{1}, \hat{\theta}_{2}$ and $\hat{\theta}_{3}$ solve

$$
\begin{aligned}
\frac{\partial l}{\partial \theta_{0}} & =n_{D}-\sum_{z=1}^{\mathcal{Z}} n_{D z} \sum_{i \in U_{z}} \frac{\exp \left\{\theta_{0}+\eta(z)+\theta_{1} Y_{i}+\theta_{2}^{T} Z_{i}^{M}+\theta_{3}^{T} Y_{i} Z_{i}^{I}\right\}}{n_{\bar{D} z}+n_{D z} \exp \left\{\theta_{0}+\eta(z)+\theta_{1} Y_{i}+\theta_{2}^{T} Z_{i}^{M}+\theta_{3}^{T} Y_{i} Z_{i}^{I}\right\}}=0, \\
\frac{\partial l}{\partial \theta_{1}} & =\sum_{z=1}^{\mathcal{Z}} \sum_{i \in U_{D z}} Y_{i}-\sum_{z=1}^{\mathcal{Z}} n_{D z} \sum_{i \in U_{z}} Y_{i} \frac{\exp \left\{\theta_{0}+\eta(z)+\theta_{1} Y_{i}+\theta_{2}^{T} Z_{i}^{M}+\theta_{3}^{T} Y_{i} Z_{i}^{I}\right\}}{n_{\bar{D} z}+n_{D z} \exp \left\{\theta_{0}+\eta(z)+\theta_{1} Y_{i}+\theta_{2}^{T} Z_{i}^{M}+\theta_{3}^{T} Y_{i} Z_{i}^{I}\right\}}=0, \\
\frac{\partial l}{\partial \theta_{2}} & =\sum_{z=1}^{\mathcal{Z}} \sum_{i \in U_{D z}} Z_{i}^{M}-\sum_{z=1}^{\mathcal{Z}} n_{D z} \sum_{i \in U_{z}} Z_{i}^{M} \frac{\exp \left\{\theta_{0}+\eta(z)+\theta_{1} Y_{i}+\theta_{2}^{T} Z_{i}^{M}+\theta_{3}^{T} Y_{i} Z_{i}^{I}\right\}}{n_{\bar{D} z}+n_{D z} \exp \left\{\theta_{0}+\eta(z)+\theta_{1} Y_{i}+\theta_{2}^{T} Z_{i}^{M}+\theta_{3}^{T} Y_{i} Z_{i}^{I}\right\}}=0, \\
\frac{\partial l}{\partial \theta_{3}}= & \sum_{z=1}^{\mathcal{Z}} \sum_{i \in U_{D z}} Y_{i} Z_{i}^{I}-\sum_{z=1}^{\mathcal{Z}} n_{D z} \sum_{i \in U_{z}} Y_{i} Z_{i}^{I} \frac{\exp \left\{\theta_{0}+\eta(z)+\theta_{1} Y_{i}+\theta_{2}^{T} Z_{i}^{M}+\theta_{3}^{T} Y_{i} Z_{i}^{I}\right\}}{n_{\bar{D} z}+n_{D z} \exp \left\{\theta_{0}+\eta(z)+\theta_{1} Y_{i}+\theta_{2}^{T} Z_{i}^{M}+\theta_{3}^{T} Y_{i} Z_{i}^{I}\right\}}=0,
\end{aligned}
$$

Observe that these are the score equations if we fit a prospective logistic model

$$
\operatorname{logit}\{P(D=1 \mid Y, Z)\}=\theta_{0}+\xi(Z)+\theta_{1} Y+\theta_{2}^{T} Z^{M}+\theta_{3}^{T} Y Z^{I}
$$

to the case-control sample using $\xi(z)=\log \left(n_{D z} / n_{\bar{D} z}\right)+\eta(z)$ as an offset term.

Therefore, to calculate the predictiveness curve specific for covariate group $z$, we first fit a prospective logistic model to the data and calculate $\hat{p}_{i z}$, the maximum likelihood estimate of $p_{i z}, i \in U_{z}$. Then we compute

$$
\begin{aligned}
& \hat{F}_{\bar{D} z}(y)=\sum_{i \in U_{z}} \hat{p}_{i z} I\left(Y_{i} \leq y\right) \\
& \hat{F}_{D z}(y)=\sum_{i \in U_{z}} \hat{p}_{i z} I\left(Y_{i} \leq y\right) \widehat{\mathcal{L R}}_{z}\left(Y_{i}\right)
\end{aligned}
$$


where $\widehat{\mathcal{L R}}_{z}$ is the MLE of $\mathcal{L} \mathrm{R}_{z}$. We estimate $F_{z}$ as a weighted average of $F_{D z}$ and $F_{\bar{D} z}$ with weight being $P(D=1 \mid Z=z)$, disease prevalence within covariate group $z$. Note $P(D=1 \mid Z=z)$ is either known or can be estimated as mentioned before. Let the estimate of $F_{z}$ be $\hat{F}_{z}(y)=P(D=1 \mid Z=z) \hat{F}_{D z}(y)+P(D=$ $0 \mid Z=z) \hat{F}_{\bar{D} z}(y)$. The semiparametric maximum likelihood estimator (MLE) of the predictiveness curve and its inverse within covariate group $z$ are:

$$
\begin{aligned}
& \hat{R}_{z}(v)=G\left\{\hat{\theta}, \hat{F}_{z}^{-1}(v), z\right\} \quad \text { for } v \in(0,1) \\
& \hat{R}_{z}^{-1}(p)=\hat{F}_{z}\left\{G^{-1}(\hat{\theta}, p, z)\right\} \quad \text { for } p \in\left\{R_{z}(v): v \in(0,1)\right\}
\end{aligned}
$$

where $G^{-1}(\theta, p, z)=\{y: G(\theta, y, z)=p\}$.

Observe that for the special case where $Z^{I}$ is also the covariate group indicator, we essentially allow for a different risk model within each covariate group, and our methods are equivalent to fitting the predictiveness curve using the semiparametric two-sample MLE method (Huang, 2007) within each covariate group separately.

Based on similar arguments as in Huang (2007), asymptotic normality of $\hat{R}_{z}(v)$ and $\hat{R}_{z}^{-1}(p)$ can be derived. Proofs of the following theorems are outlined in the appendix.

\section{Theorem 1}

As $n \rightarrow \infty, \sqrt{n}\left\{\hat{R}_{z}(v)-R_{z}(v)\right\}$ converges to a normal random variable with mean zero and variance

$$
\begin{aligned}
\Sigma_{1 z}(v) & =\left\{\frac{\partial R_{z}(v)}{\partial v}\right\}^{2} \operatorname{var}\left(\sqrt{n}\left[\hat{F}_{z}\left\{F_{z}^{-1}(v)\right\}-v\right]\right)+\left(\frac{\partial R_{z}(v)}{\partial \theta}\right)^{T} \operatorname{var}\{\sqrt{n}(\hat{\theta}-\theta)\}\left(\frac{\partial R_{z}(v)}{\partial \theta}\right) \\
& +2\left(\frac{\partial R_{z}(v)}{\partial \theta}\right)^{T} \operatorname{cov}\left(\sqrt{n}(\hat{\theta}-\theta), \sqrt{n}\left[\hat{F}_{z}\left\{F_{z}^{-1}(v)\right\}-v\right]\right)\left\{\frac{\partial R_{z}(v)}{\partial v}\right\} .
\end{aligned}
$$

\section{Theorem 2}

As $n \rightarrow \infty, \sqrt{n}\left\{\hat{R}_{z}^{-1}(p)-R_{z}^{-1}(p)\right\}$ converges to a normal random variable with mean zero and variance

$$
\begin{aligned}
\Sigma_{2 z}(p) & =\operatorname{var}\left(\sqrt{n}\left[\hat{F}_{z}\left\{G^{-1}(\theta, p, z)\right\}-F_{z}\left\{G^{-1}(\theta, p, z)\right\}\right]\right) \\
& +\left(\frac{\partial R_{z}^{-1}(p)}{\partial \theta}\right)^{T} \operatorname{var}\{\sqrt{n}(\hat{\theta}-\theta)\}\left(\frac{\partial R_{z}^{-1}(p)}{\partial \theta}\right) \\
& +2\left(\frac{\partial R_{z}^{-1}(p)}{\partial \theta}\right)^{T} \operatorname{cov}\left(\sqrt{n}(\hat{\theta}-\theta), \sqrt{n}\left[\hat{F}_{z}\left\{G^{-1}(\theta, p, z)\right\}-F_{z}\left\{G^{-1}(\theta, p, z)\right\}\right]\right) .
\end{aligned}
$$


Observe that when $v=R_{z}^{-1}(p)$,

$$
\frac{\partial R_{z}^{-1}(p)}{\partial \theta} \frac{\partial R_{z}(v)}{\partial v}=\frac{\partial v}{\partial \theta} \frac{\partial R_{z}(v)}{\partial v}=\frac{\partial R_{z}(v)}{\partial \theta},
$$

thus $\Sigma_{1 z}(v)=\left\{\partial R_{z}(v) / \partial v\right\}^{2} \Sigma_{2 z}(p)$. That is the variance of $\hat{R}_{z}(v)$ and its inverse are related by a factor equal to square of the derivative of $R_{z}(v)$, which is intuitive since a perturbation in $R_{z}(v)$ can be approximated by $R_{z}(v)$ times a perturbation in $R_{z}^{-1}(p)$. In practice, bootstrap resampling is employed to assess sampling variability, avoiding nonparametric density estimation.

\subsection{Continuous Covariate}

In Section 2.1, we developed an estimator of the covariate-specific predictiveness curve from a matched case-control study when covariates of interest are discrete. In this section, we investigate estimation in more general settings where continuous covariates can be involved.

Our strategy is to separate the estimation of the covariate-specific predictiveness curve into estimation of the risk model and estimation of the covariate-specific marker distribution. The first estimation can be achieved employing logistic regression model adjusted by the known disease prevalences within the matching strata. The second estimation can be performed using a weighted version of the semiparametric location-scale model (Heagerty and Pepe, 1999) for the marker distribution.

Consider the general risk model in (1). We apply a logistic model

$$
\operatorname{logit}\{P(D=1 \mid Y, Z, S=s)\}=\theta_{0}+\xi(s)+\kappa\left(\theta_{1}, Y, Z\right)
$$

to the case-control sample using

$$
\xi(s)=\log \left\{\frac{P(D=1 \mid S=s, \text { sampled })}{P(D=0 \mid S=s, \text { sampled })} \frac{1-\rho_{s}}{\rho_{s}}\right\}
$$

as the offset term, where $P(D=1 \mid S=s$, sampled $) / P(D=0 \mid S=s$, sampled $)$ is fixed by design and/or can be estimated in the case-control sample with $n_{D s} / n_{\bar{D} s}$.

Fitting this modified prospective logistic regression is essentially maximizing the "conditional (or pseudo) maximum likelihood" that an observation in the case-control sample within a particular stratum is a case or a control (Manski and Fadden, 1981; Hsieh et al., 1985; Breslow and Cain, 1988; Fears and Brown, 1986; Breslow and Zhao, 1988; Scott and Wild, 2001). Specifically, the contribution of subject $i$ to the 
pseudolikelihood is $P\left(D_{i} \mid Y_{i}, Z_{i}, S_{i}\right.$, sampled). According to Bayes' theorem, the odds of being diseased in the case-control sample is

$$
\begin{aligned}
& \frac{P(D=1 \mid Y, Z, S, \text { sampled })}{P(D=0 \mid Y, Z, S, \text { sampled })}=\frac{P(Y, Z \mid D=1, S, \text { sampled })}{P(Y, Z \mid D=0, S, \text { sampled })} \frac{P(D=1 \mid S, \text { sampled })}{P(D=0 \mid S, \text { sampled })} \\
= & \frac{P(Y, Z \mid D=1, S)}{P(Y, Z \mid D=0, S)} \frac{P(D=1 \mid S, \text { sampled })}{P(D=0 \mid S, \text { sampled })} \\
= & \frac{P(D=1 \mid Y, Z, S) P(D=0 \mid S)}{P(D=0 \mid Y, Z, S) P(D=1 \mid S)} \frac{P(D=1 \mid S, \text { sampled })}{P(D=0 \mid S, \text { sampled })} \\
= & \frac{P(D=1 \mid Y, Z) P(D=0 \mid S)}{P(D=0 \mid Y, Z) P(D=1 \mid S)} \frac{P(D=1 \mid S, \text { sampled })}{P(D=0 \mid S, \text { sampled })} \\
= & \exp \left\{\theta_{0}+\xi(S)+\kappa\left(\theta_{1}, Y, Z\right)\right\} .
\end{aligned}
$$

Note that when the covariate of interest is the matching stratum, this pseudolikelihood approach leads to the same estimates of the risk model as in the MLE approach in Section 2.1.

For a more complicated design, the two-phase design, this pseudolikelihood method has been shown to be pretty similar to the maximum likelihood estimator which can be obtained by repeated fitting of ordinary logistic regression models (Wild, 1991; Scott and Wild, 1997; Breslow and Holubkov, 1997). Both estimators are shown to be much more efficient than the inverse probability weighted likelihood approach (Flanders and Greenland, 1991).

To estimate $F_{z}$, we employ a semiparametric location-scale model for the distribution of $Y$ given $Z$ (Heagerty and Pepe, 1999). Suppose

$$
F_{z}(y)=F_{0}\left(\frac{y-\mu_{z}}{\sigma_{z}}\right),
$$

where $F_{0}$ is the cumulative distribution function of some unknown distribution, $\mu_{Z}=\gamma^{T} U(Z)$ and $\log \left(\sigma_{Z}\right)=$ $\delta^{T} W(Z)$, and $U(Z)$ and $W(Z)$ are specified functions of $Z$. For example, for a discrete $Z, U(Z)$ and $W(Z)$ could be dummy variables indicating unique values of $Z$, while for a continuous $Z$, they could be B-spline basis for $Z$. Denote $U_{i}=U\left(Z_{i}\right)$ and $W_{i}=W\left(Z_{i}\right)$. For a cohort study, Heagerty and Pepe (1999) proposed estimating $\gamma$ and $\delta$ by solving the estimating equations

$$
\begin{aligned}
& \sum_{i=1}^{n} U_{i}\left(Y_{i}-\gamma^{T} U_{i}\right) / \sigma_{Z_{i}}^{2}=0 \\
& \sum_{i=1}^{n} W_{i}\left\{\left(Y_{i}-\gamma^{T} U_{i}\right)^{2}-\sigma_{Z_{i}}^{2}\right\} / \sigma_{Z_{i}}^{2}=0 .
\end{aligned}
$$

For the matched case-control study, we obtain $\hat{\gamma}$ and $\hat{\delta}$ as solutions to the inverse probability weighted 
(Horvitz and Thompson, 1952) version of these estimating equations in (9):

$$
\begin{aligned}
& \sum_{i=1}^{n} \frac{1}{\hat{q}_{i}} U_{i}\left(Y_{i}-\gamma^{T} U_{i}\right) / \sigma_{Z_{i}}^{2}=0 \\
& \sum_{i=1}^{n} \frac{1}{\hat{q}_{i}} W_{i}\left\{\left(Y_{i}-\gamma^{T} U_{i}\right)^{2}-\sigma_{Z_{i}}^{2}\right\} / \sigma_{Z_{i}}^{2}=0
\end{aligned}
$$

where $\hat{q}_{i}=\hat{P}\left(\right.$ sampled $\left.\mid Y_{i}, Z_{i}, S_{i}, D_{i}\right)$. Observe that

$$
P\left(\text { sampled } \mid Y_{i}, Z_{i}, S_{i}, D_{i}\right)=P\left(\text { sampled } \mid S_{i}, D_{i}\right)=\frac{P\left(D_{i} \mid S_{i}, \text { sampled }\right) P\left(S_{i} \mid \text { sampled }\right)}{P\left(D_{i} \mid S_{i}\right) P\left(S_{i}\right)}
$$

where $P\left(D_{i} \mid S_{i}\right.$, sampled $)$ is fixed by design and can be estimated as well. So if $P\left(S_{i}\right)$ is known in addition to $P\left(D_{i} \mid S_{i}\right)$, we can estimate $P\left(S_{i} \mid\right.$ sampled $)$ from the case-control sample and plug in

$$
\hat{q}_{i}=\frac{\hat{P}\left(D_{i} \mid S_{i}, \text { sampled }\right) \hat{P}\left(S_{i} \mid \text { sampled }\right)}{P\left(D_{i} \mid S_{i}\right) P\left(S_{i}\right)}
$$

Furthermore, we estimate $F_{0}$ by

$$
\hat{F}_{0}(c)=\sum_{i=1}^{n} \frac{1}{\hat{q}_{i}} I\left(\frac{Y_{i}-\hat{\gamma}^{T} U_{i}}{e^{\hat{\delta}^{T} W_{i}}} \leq c\right) / \sum_{i=1}^{n} \frac{1}{\hat{q}_{i}}
$$

The covariate-specific marker distribution estimate is

$$
\hat{F}_{z}(y)=\hat{F}_{0}\left(\frac{y-\hat{\gamma}^{T} u}{e^{\hat{\delta}^{T} w}}\right)
$$

where $u=U(z)$ and $w=W(z)$. The corresponding $v^{t h}$ quantile is

$$
\hat{F}_{z}^{-1}(v)=\hat{\gamma}^{T} u+e^{\hat{\delta}^{T} w} \hat{F}_{0}^{-1}(v) \quad \text { for } v \in(0,1)
$$

Estimators of the covariate-specific predictiveness curve for $Y$ given $Z=z$ which we call PMLE are

$$
\begin{aligned}
& \hat{R}_{z}(v)=G\left\{\hat{\theta}, \hat{F}_{z}^{-1}(v), z\right\} \quad \text { for } v \in(0,1) \\
& \hat{R}_{z}^{-1}(p)=\hat{F}_{z}\left\{G^{-1}(\hat{\theta}, p, z)\right\} \quad \text { for } p \in\left\{R_{z}(v): v \in(0,1)\right\} .
\end{aligned}
$$

Note that compared to the MLE method in Section 2.1, the PMLE method requires additional auxiliary information about the distribution of $S$. If the distribution of $Y$ conditional on $Z$ is independent of the matching stratum $S$, we can modify the PMLE method such that the extra piece of information about $P(S)$ is not necessary. This is satisfied in current setting since $S$ can be represented as a function of $Z$. The concept is to ensure unbiasedness of these estimating equations in (10) averaged across the distribution of $Y$ within each stratum rather than averaged over the general population. 
This way it is sufficient to adjust for selection bias within each stratum. For any two subjects $i, j$ in the same stratum, we want to choose $q_{i}$ and $q_{j}$ such that

$$
q_{i} / q_{j}=P\left(\operatorname{sampled} \mid D_{i}, Y_{i}, Z_{i}, S_{i}\right) / P\left(\operatorname{sampled} \mid D_{j}, Y_{j}, Z_{j}, S_{j}\right)
$$

Given $S_{i}=S_{j}$, we need to have

$$
\begin{aligned}
& \frac{P\left(\text { sampled } \mid D_{i}, Y_{i}, Z_{i}, S_{i}\right)}{P\left(\text { sampled } \mid D_{j}, Y_{j}, Z_{j}, S_{j}\right)}=\frac{P\left(\text { sampled } \mid D_{i}, S_{i}\right)}{P\left(\text { sampled } \mid D_{j}, S_{j}\right)} \\
= & \frac{P\left(D_{i}, S_{i} \mid \text { sampled }\right) P\left(D_{j}, S_{j}\right)}{P\left(D_{j}, S_{j} \mid \text { sampled }\right) P\left(D_{i}, S_{i}\right)}=\frac{P\left(D_{i} \mid S_{i}, \text { sampled }\right) P\left(D_{j} \mid S_{j}\right)}{P\left(D_{j} \mid S_{j}, \text { sampled }\right) P\left(D_{i} \mid S_{i}\right)}
\end{aligned}
$$

to guarantee the unbiasedness of (10) within each stratum. One of the choices of $q_{i}$ is $P\left(D_{i} \mid S_{i}\right.$, sampled $) P(1-$ $\left.D_{i} \mid S_{i}\right)$. We plug $\hat{q}_{i}=\hat{P}\left(D_{i} \mid S_{i}\right.$, sampled $) P\left(1-D_{i} \mid S_{i}\right)$ into (10).

The covariate-specific predictiveness curve for marker $Y$ can be derived likewise. We call the estimator PMLE-M. Note that PMLE and PMLE-M are equivalent when there is no matching in the case-control design. We use bootstrap resampling for variance estimation for both PMLE and PMLE-M estimators.

\section{Simulation Studies}

We evaluate our methodology using two simulation settings. In the first setting, cases and controls are frequency matched according to a binary covariate $Z$, which is also the covariate of interest for evaluating the marker's predictiveness. Marker $Y$ conditional on $D$ and $Z$ is normally distributed,

$$
\begin{aligned}
Y_{D} \mid Z=0 \sim N(0.5,1), & Y_{\bar{D}} \mid Z=0 \sim N(0,1), \\
Y_{D} \mid Z=1 \sim N(1,1), & Y_{\bar{D}} \mid Z=1 \sim N(0.5,1) .
\end{aligned}
$$

In the population, $Z$ takes value 1 with probability 0.5 . Disease prevalence given $Z=0,1$ are 0.1 and 0.2 respectively. Within each matching stratum defined by the value of $Z$, equal number of cases and controls are sampled, and sample sizes are constant across strata. We compare the three estimators of the covariate $Z$-specific predictiveness curves developed in Section 2: the semiparametric MLE method assuming risk is linear logistic in $Y$ and $Z$, and the PMLE and PMLE-M methods where location and log-scale parameters for the marker distribution are modeled as linear in $Z$.

In the second setting, we have a continuous covariate $Z$. The overall prevalence of $D$ is 0.15 . We have $Y$ 
Table 1: Performance of the covariate-specific predictiveness curve estimators for the first simulation setting in Section 3. Shown are results for $\hat{R}_{z}(v)$ with $z=0$.

\begin{tabular}{|c|c|c|c|c|c|c|}
\hline & & $v=0.1$ & $v=0.3$ & $v=0.5$ & $v=0.7$ & $v=0.9$ \\
\hline & $R_{z}(v)$ & 0.05 & 0.072 & 0.091 & 0.116 & 0.161 \\
\hline \multicolumn{7}{|l|}{ Bias } \\
\hline \multicolumn{7}{|c|}{$\%$ bias in $\hat{R}_{z}(v)$} \\
\hline \multirow{3}{*}{$n=200$} & MLE & 1.76 & -0.31 & -0.56 & -0.59 & 0.06 \\
\hline & PMLE-M & 1.76 & -0.5 & -0.71 & -0.78 & 0.25 \\
\hline & PMLE & 1.67 & -0.42 & -0.78 & -0.74 & 0.08 \\
\hline \multirow[t]{3}{*}{$n=500$} & MLE & 0.4 & -0.37 & -0.41 & -0.26 & 0.32 \\
\hline & PMLE-M & 0.39 & -0.33 & -0.19 & -0.18 & 0.39 \\
\hline & PMLE & 0.3 & -0.3 & -0.23 & -0.16 & 0.36 \\
\hline \multirow[t]{3}{*}{$n=1200$} & MLE & -0.26 & -0.22 & -0.18 & -0.16 & 0.22 \\
\hline & PMLE-M & -0.09 & -0.29 & -0.22 & -0.2 & 0.26 \\
\hline & PMLE & -0.07 & -0.28 & -0.23 & -0.19 & 0.26 \\
\hline \multicolumn{7}{|c|}{ MSE Efficiency relative to MLE } \\
\hline \multirow[t]{2}{*}{$n=200$} & PMLE-M & 0.98 & 0.98 & 0.92 & 0.68 & 0.87 \\
\hline & PMLE & 0.99 & 0.99 & 0.94 & 0.71 & 0.9 \\
\hline \multirow[t]{2}{*}{$n=500$} & PMLE-M & 1.01 & 0.99 & 0.92 & 0.77 & 0.86 \\
\hline & PMLE & 1.01 & 1 & 0.96 & 0.82 & 0.86 \\
\hline \multirow[t]{2}{*}{$n=1200$} & PMLE-M & 0.98 & 0.96 & 0.95 & 0.68 & 0.87 \\
\hline & PMLE & 0.98 & 0.98 & 0.96 & 0.74 & 0.87 \\
\hline \multicolumn{7}{|c|}{$\begin{array}{l}95 \% \text { Percentile Bootstrap CI } \\
\text { coverage }(\%)\end{array}$} \\
\hline \multirow[t]{3}{*}{$n=200$} & MLE & 92.2 & 93.5 & 95.4 & 97.9 & 94 \\
\hline & PMLE-M & 92.3 & 94.7 & 96.5 & 96.9 & 94.1 \\
\hline & PMLE & 92.2 & 94.8 & 96.5 & 96 & 94.3 \\
\hline \multirow[t]{3}{*}{$n=500$} & MLE & 94.6 & 93.9 & 95.2 & 96.2 & 94.6 \\
\hline & PMLE-M & 94.3 & 94 & 95.7 & 96.6 & 94.6 \\
\hline & PMLE & 94.4 & 93.8 & 95.6 & 96.1 & 94.8 \\
\hline \multirow[t]{3}{*}{$n=1000$} & MLE & 94 & 94.1 & 94.7 & 95.1 & 94.6 \\
\hline & PMLE-M & 93.8 & 93.9 & 93.3 & 95 & 94.5 \\
\hline & PMLE & 94.1 & 93.9 & 93.9 & 95.1 & 94.2 \\
\hline
\end{tabular}

and $Z$ bivariate normally distributed with correlation 0.5 conditional on $D$,

$$
\begin{aligned}
& \left(\begin{array}{c}
Y \\
Z
\end{array}\right) \mid D=0 \sim N\left(\left(\begin{array}{l}
0 \\
0
\end{array}\right),\left(\begin{array}{cc}
1 & 0.5 \\
0.5 & 1
\end{array}\right)\right), \\
& \left(\begin{array}{c}
Y \\
Z
\end{array}\right) \mid D=1 \sim N\left(\left(\begin{array}{l}
1 \\
0
\end{array}\right),\left(\begin{array}{cc}
1 & 0.5 \\
0.5 & 1
\end{array}\right)\right) .
\end{aligned}
$$

During the simulation cases are randomly sampled from the case population and for each case, a control with the same $Z$ value is generated. We again compare the three estimators of covariate $Z$-specific predictiveness curves. For the semiparametric MLE estimator, risk is modeled as linear logistic in $Y$ and discretized $Z$ (where cutoff points are chosen to be quintiles of $Z$ in the case population). For the PMLE and PMLE-M estimators, location and $\log ($ scale) parameters for the marker distribution are modeled as linear in $Z$.

For sample size varying from 200 to 1200 , we evaluate estimates of $R_{z}(v)$ and $R_{z}^{-1}(p)$ for $v=0.1,0.3,0.5,0.7,0.9$ and corresponding $p=R_{z}(v)$.

For the first setting, simulation results for $Z=0$ are presented in Tables 1 and 2. For the second setting, simulation results for $Z$ equal to the median in the case population are presented in Tables 3 and 4 . We find that in both settings the three estimators have reasonably good performance. Bias was minimal for $R_{z}(v)$ and for $R_{z}^{-1}(p)$. Coverage of the $95 \%$ confidence intervals constructed from the bootstrap distribution 
Table 2: Performance of the covariate-specific predictiveness curve estimators for the first simulation setting in Section 3. Shown are results for $\hat{R}_{z}^{-1}(p)$ with $z=0$.

\begin{tabular}{|c|c|c|c|c|c|c|}
\hline \multirow{3}{*}{ Bias } & \multirow{3}{*}{$R_{z}^{-1}(p)$} & $p=0.05$ & \multirow{2}{*}{$\begin{array}{c}p=0.072 \\
0.30\end{array}$} & \multirow{3}{*}{$\begin{array}{c}p=0.091 \\
0.50\end{array}$} & \multirow{3}{*}{$\begin{array}{c}p=0.116 \\
0.70\end{array}$} & \multirow{2}{*}{$\begin{array}{c}p=0.161 \\
0.90\end{array}$} \\
\hline & & 0.10 & & & & \\
\hline & & & & & & \\
\hline \multicolumn{7}{|c|}{$\%$ bias in $\hat{R}_{z}^{-1}(p)$} \\
\hline \multirow[t]{3}{*}{$n=200$} & MLE & 9.11 & -4.66 & -2.26 & 1.6 & 0.67 \\
\hline & PMLE-M & 9.99 & -4.48 & -1.84 & 1.93 & 0.65 \\
\hline & PMLE & 9.98 & -4.41 & -1.83 & 1.91 & 0.68 \\
\hline \multirow[t]{3}{*}{$n=500$} & MLE & 4.23 & -1.06 & -0.34 & 0.51 & 0.17 \\
\hline & PMLE-M & 4.55 & -1.23 & -0.61 & 0.48 & 0.18 \\
\hline & PMLE & 4.68 & -1.3 & -0.6 & 0.47 & 0.19 \\
\hline \multirow{3}{*}{$n=1200$} & MLE & 3.12 & -0.25 & -0.22 & 0.28 & 0.04 \\
\hline & PMLE-M & 2.78 & -0.2 & -0.06 & 0.33 & 0.05 \\
\hline & PMLE & 2.76 & -0.18 & -0.04 & 0.33 & 0.04 \\
\hline \multicolumn{7}{|c|}{ MSE Efficiency relative to MLE } \\
\hline \multirow[t]{2}{*}{$n=200$} & PMLE-M & 1 & 0.98 & 0.92 & 0.77 & 0.9 \\
\hline & PMLE & 1.01 & 0.98 & 0.92 & 0.79 & 0.91 \\
\hline \multirow[t]{2}{*}{$n=500$} & PMLE-M & 1.01 & 0.98 & 0.89 & 0.82 & 0.89 \\
\hline & PMLE & 1.02 & 0.98 & 0.9 & 0.88 & 0.89 \\
\hline \multirow[t]{2}{*}{$n=1200$} & PMLE-M & 0.98 & 0.96 & 0.94 & 0.71 & 0.89 \\
\hline & PMLE & 0.98 & 0.96 & 0.96 & 0.74 & 0.89 \\
\hline \multicolumn{7}{|c|}{$\begin{array}{l}\text { 95\% Percentile Bootstrap CI } \\
\text { coverage }(\%)\end{array}$} \\
\hline \multirow[t]{3}{*}{$n=200$} & MLE & 92.1 & 93.5 & 95.5 & 97.9 & 93.9 \\
\hline & PMLE-M & 92.2 & 94.9 & 96.6 & 97 & 94 \\
\hline & PMLE & 92.3 & 94.7 & 96.7 & 96.1 & 94.5 \\
\hline \multirow[t]{3}{*}{$n=500$} & MLE & 94.5 & 94 & 95.3 & 96.3 & 94.6 \\
\hline & PMLE-M & 94.2 & 93.9 & 95.7 & 96.6 & 94.6 \\
\hline & PMLE & 94.5 & 93.6 & 95.7 & 96.1 & 94.8 \\
\hline \multirow[t]{3}{*}{$n=1200$} & MLE & 94 & 94.2 & 94.7 & 95.2 & 94.6 \\
\hline & PMLE-M & 93.8 & 93.9 & 93.3 & 95.1 & 94.5 \\
\hline & PMLE & 94.1 & 94 & 93.9 & 95.2 & 94.2 \\
\hline
\end{tabular}

Table 3: Performance of the covariate-specific predictiveness curve estimators for the second simulation setting in Section 3. Shown are results for $\hat{R}_{z}(v)$ with $z$ being the median of $Z$ in the case population.

\begin{tabular}{|c|c|c|c|c|c|c|}
\hline & & $v=0.1$ & $v=0.3$ & $v=0.5$ & $v=0.7$ & $\bar{v}=0.9$ \\
\hline & $R_{z}(v)$ & 0.022 & 0.054 & 0.097 & 0.173 & 0.358 \\
\hline \multicolumn{7}{|l|}{ Bias } \\
\hline \multicolumn{7}{|c|}{$\%$ bias in $\hat{R}_{z}(v)$} \\
\hline \multirow{3}{*}{$n=200$} & MLE & 12.21 & 3.85 & 0.01 & -0.72 & 0.14 \\
\hline & PMLE-M & 3.53 & 0.63 & -1.24 & -0.4 & -0.06 \\
\hline & PMLE & 3.87 & 0.91 & -0.88 & -0.18 & 0.04 \\
\hline \multirow[t]{3}{*}{$n=500$} & MLE & 7 & 2.77 & 0.58 & 0.14 & -0.63 \\
\hline & PMLE-M & 0.22 & -0.58 & -0.81 & -0.22 & 0.52 \\
\hline & PMLE & -0.05 & -0.7 & -0.91 & -0.34 & 0.61 \\
\hline \multirow[t]{3}{*}{$n=1200$} & MLE & 7.24 & 3.97 & 1.89 & 0.22 & -1.04 \\
\hline & PMLE-M & 0.36 & -0.13 & -0.36 & -0.09 & 0.13 \\
\hline & PMLE & 0.33 & -0.19 & -0.3 & -0.08 & 0.06 \\
\hline \multicolumn{7}{|c|}{ MSE Efficiency relative to MLE } \\
\hline \multirow[t]{2}{*}{$n=200$} & PMLE-M & 1.77 & 1.61 & 2.03 & 2.86 & 1.81 \\
\hline & PMLE & 1.8 & 1.58 & 1.82 & 2.12 & 1.51 \\
\hline \multirow[t]{2}{*}{$n=500$} & PMLE-M & 1.98 & 1.82 & 1.84 & 2.75 & 1.76 \\
\hline & PMLE & 1.92 & 1.77 & 1.68 & 2 & 1.41 \\
\hline \multirow[t]{2}{*}{$n=1200$} & PMLE-M & 2.19 & 2.02 & 2.01 & 2.69 & 1.78 \\
\hline & PMLE & 2.12 & 1.95 & 1.81 & 2.01 & 1.54 \\
\hline \multicolumn{7}{|c|}{$\begin{array}{l}\text { 95\% Percentile Bootstrap CI } \\
\text { coverage }(\%)\end{array}$} \\
\hline \multirow[t]{3}{*}{$n=200$} & MLE & 96.1 & 96.8 & 97.2 & 98.7 & 98.7 \\
\hline & PMLE-M & 94.3 & 94.2 & 94.2 & 98.2 & 96.9 \\
\hline & PMLE & 93 & 91.6 & 92.4 & 95.5 & 97.9 \\
\hline \multirow[t]{3}{*}{$n=500$} & MLE & 95.1 & 95.5 & 96.2 & 98.8 & 96.9 \\
\hline & PMLE-M & 93.8 & 93.7 & 93.5 & 97.7 & 95.1 \\
\hline & PMLE & 92.3 & 91.9 & 90.9 & 95.7 & 95.9 \\
\hline \multirow[t]{3}{*}{$n=1200$} & MLE & 94.8 & 94 & 94.7 & 97.5 & 95.8 \\
\hline & PMLE-M & 94.2 & 94.1 & 94.9 & 96.2 & 95.7 \\
\hline & PMLE & 93.3 & 91.8 & 91.1 & 93 & 96.6 \\
\hline
\end{tabular}


Table 4: Performance of the covariate-specific predictiveness curve estimators for the second simulation setting in Section 3. Shown are results for $\hat{R}_{z}^{-1}(p)$ with $z$ being the median of $Z$ in the case population.

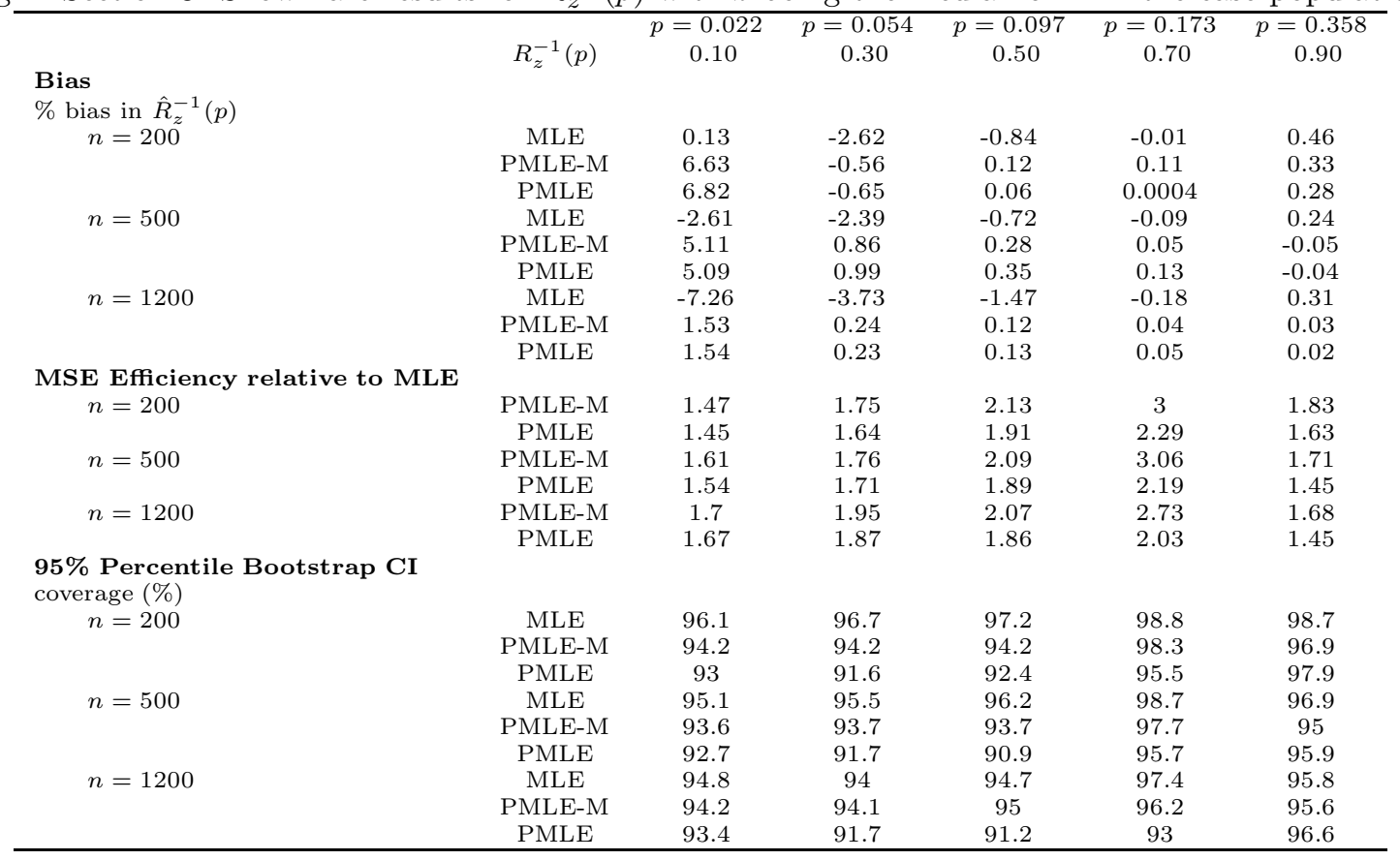

is fairly close to the nominal level. In the first setting where there are two covariate groups, MSE for the three estimators are similar in magnitude. The MLE estimator is slightly more efficient than the PMLE and PMLE-M estimators for certain $v$. As the number of (discretized) covariate groups increases to five (the second setting), the MLE estimator becomes much less efficient compared to the PMLE and PMLE-M estimators.

Since the MLE method requires less model assumption, it is more robust than the PMLE (PMLE-M) methods. In practice the MLE method is preferred when the number of covariate groups is relatively small and the number of observations in each group is not too small, e.g. when covariate is baseline risk category in the renal example. On the other hand, since the PMLE (PMLE-M) methods borrow information across covariate groups during estimation of the marker distribution, it is expected to be more efficient compared to the MLE method as the number of covariate groups increases. Specifically, when we have several continuous covariates, like those baseline covariate values described in the renal example, the job to discretize these covariates for MLE estimation becomes very difficult and the PMLE (PMLE-M) methods are more appealing in this scenario. 


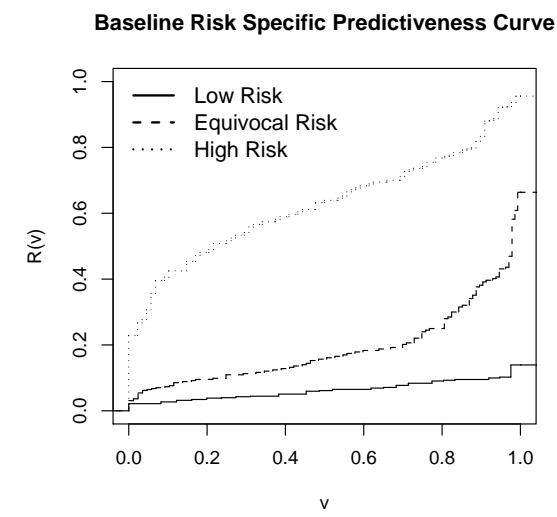

Figure 1: Predictiveness curves for serum creatinine specific to patients with low, high, and equivocal baseline risk.

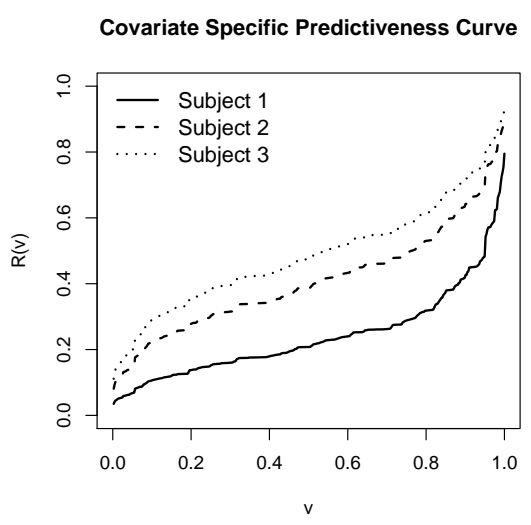

Figure 2: Examples of covariate-specific predictiveness curves for serum creatinine.

\section{Illustration}

We illustrate the methodology evaluating serum creatinine as a risk prediction marker for renal artery stenosis in patients with therapy resistant hypertension. The original cohort consists of 426 hypertensive patients undergoing renal angiography (Janssens et al., 2005; Krijnen et al., 1998). Baseline risk is modeled with age, smoking status (ever) and their interaction, gender, hypertension, BMI, abdominal bruit, atherosclerosis disease, and hypercholesterolaemia. Consider a low risk threshold of 0.1 below which no routine angiography is recommended and a high risk threshold of 0.4 above which routine angiography is encouraged. There are 162 subjects in the cohort with low baseline risk (10 cases), 176 subjects with medium baseline risk (33 cases), and 88 subjects with high baseline risk (55 cases). A stratified case-control sample of size 217 is 
generated including all 98 cases and controls frequency matched according to baseline risk group. Within low and medium baseline risk strata, the number of controls selected are twice that of cases, while within the high risk group, all controls are selected.

Figure 1 displays the predictiveness curves for serum creatinine, for patients within different baseline risk strata. Hence the covariate is baseline risk stratification, the same as the matching stratum. The MLE method was employed in estimation. Observe that for those patients who have low baseline risks, after measuring serum creatinine, there is $\hat{R}_{z}^{-1}(0.1)=94.5 \%$ chance that they will remain classified as low risk (with $95 \%$ CI $(94.8 \%, 100 \%)$ ), a small chance that their risk will be elevated to the intermediate risk range $\left(\hat{R}_{z}^{-1}(0.4)-\hat{R}_{z}^{-1}(0.1)=5.2 \%\right.$ with $95 \%$ CI $\left.(0,24.0 \%)\right)$, and a negligible chance that their estimates risks will be high enough to receive treatment recommendation $\left(1-\hat{R}_{z}^{-1}(0.4)=0\right.$ with $95 \%$ CI $\left.(0,0)\right)$. For those patients who are originally in the medium risk range, after measuring serum creatinine, they have $24.8 \%$ chance of being reclassified as low risk (with $95 \%$ CI $(2.3 \%, 42.8 \%)$ ), $7.1 \%$ chance of being reclassified as high risk (with $95 \%$ CI $(0.8 \%, 14.6 \%)$, and $68.1 \%$ chance of remaining in the risk grey zone $(95 \%$ CI $(43.4 \%$, 95.4\%)). For those patients whose baseline risk is high, if their serum creatinine levels are measured, their estimated risks have $90.9 \%$ chance of remaining high (with $95 \%$ CI $(80.7 \%, 97.7 \%)), 9.1 \%$ chance of being medium (with $95 \%$ CI $(2.3,19.3 \%)$ ), and almost zero chance of being low enough such that treatment is deemed unnecessary (with $95 \%$ CI $(0,0)$ ). Note that for individuals originally in the medium risk range, measuring serum creatinine makes it easier for physicians to make recommendation about their subsequent medical procedures, whereas it is the other way around for those with low or high baseline risks.

For individual patients, detailed information about one's baseline covariate values is more informative than the risk group one belongs to. Not only do individual baseline risks vary within a baseline risk category but also the new marker's distribution may depend on the baseline covariates. Whenever information about baseline covariates are available, the covariate-specific predictiveness curve can be used to tailor a patient's view about worthiness of having an extra test. To illustrate this, we display covariates-specific predictiveness curves for three individuals using the PMLE method (Figure 2). Results based on PMLE-M method are fairly similar (omitted). Subject 1 is a 57 years old female with BMI $26.8 \mathrm{~kg} / \mathrm{m}^{3}$ who has a smoking history, hypertension and hypercholesterolaemia. Her baseline risk is $25.6 \%$. Subject 2 is a 65 years old male with BMI $26.0 \mathrm{~kg} / \mathrm{m}^{3}$ who has atherosclerosis disease. His baseline risk is $38.1 \%$. Subject 3 is a 57 years old female 
with BMI $22.2 \mathrm{~kg} / \mathrm{m}^{3}$ who has smoking history and atherosclerosis disease. Her baseline risk is $42.6 \%$. Next we look closely at the impact of measuring serum creatinine on each patient.

Subject 1 has her baseline risk in the middle of the equivocal range. If serum creatinine is included in the risk calculation, she has $8.6 \%$ chance of being reclassified as low risk (with $95 \%$ CI $(0 \%, 50.1 \%)), 11.9 \%$ chance $(95 \%$ CI $(2.6 \%, 47.0 \%))$ of being reclassified as high risk, and $79.5 \%$ chance $(95 \%$ CI $(36.8,89.0 \%))$ of remaining in the equivocal zone. Subject 2 is originally at the high end of the equivocal risk range. If his serum creatinine level is measured, he has $0.5 \%$ chance of being declared as low risk (with $95 \%$ CI $(0 \%$, $10.7 \%)), 50.7 \%$ chance $(95 \%$ CI $(10.5 \%, 84.5 \%))$ of remaining in the risk grey zone. At the same time, there is $50.7 \%$ chance $(95 \% \mathrm{CI}(10.5 \%, 84.5 \%))$ that he will be classified as high risk and receive recommendation for treatment. Subject 3 has her baseline risk marginally above high risk threshold. By measuring serum creatinine, there is almost zero possibility (with $95 \%$ CI $(0,5.6 \%)$ ) that she will be classified as low risk; there is $69.4 \%$ chance $(95 \%$ CI $(25.6 \%, 95.0 \%))$ that her risk will remain high and $30.6 \%$ chance $(95 \%$ CI $(25.6 \%$, 95.0\%)) that her risk will be deemed inadequate for making recommendation for or against treatment.

A subject's choice about whether or not to measure a new marker will be affected by the marker's potential impact on medical decisions as can be seen from the subject's covariate-specific predictiveness curve. The specific risk thresholds on the curve pertinent to an individual will depend on beneficial and side effects of treatment, as well as one's tolerance for the risk of the disease. In addition, the cost of measuring the raw marker may enter. For example, if subject 3 has a low tolerance for risk of disease and is willing to have routine angiography unless her risk is below $10 \%$, she might choose not to measure serum creatinine since it is highly unlikely that her estimated risk will go below $10 \%$ based on the extra test. For subject 2 , if he has low tolerance for risk, he might also choose not to measure serum creatinine since the chance for his estimated risk to drop below $10 \%$ is small; but on the other hand, if he has relatively high tolerance for risk and is willing to go through renal angiography only if his risk is above $40 \%$, then measuring serum level might be attractive to him considering the large possibility that he will have a risk calculated that allows him to decide with confidence to have renal angiography. 


\section{Discussion}

In this article we developed methodology for estimating the covariate-specific predictiveness curve for a single marker in a case-control study. Our method accommodate matching in design. Two types of semiparametric approaches are examined assuming risk is monotone increasing in the marker value. The first approach is based on empirical likelihood of $Y$ and is applicable to discrete covariates. The method can be easily generalized to the setting when $Y$ is multivariate by modeling the empirical likelihood of $\operatorname{Risk}_{z}(Y)$ following similar arguments as in Huang (2007). Note that another related estimator can be constructed by estimating $F_{D z}$ and $F_{\bar{D} z}$ empirically using the case or control sample within the $z^{t h}$ covariate group. Since the exponential tilt relationship (2) implied by the risk model is not incorporated into estimation of the marker distribution, this estimator is probably less efficient than the MLE. This has been demonstrated for the overall predictiveness curve estimated with a case-control study (Huang, 2007).

The second approach combines estimation of the risk model with estimation of the covariate-specific marker distribution assuming a semiparametric location-scale model for $Y$. Different weighting schemes can be applied to accommodate the biased sampling design. This approach is applicable to general settings and is analogous to the semiparametric approach proposed in Huang et al. (2007) for a cohort design. For a generalization of this method when $Y$ is multivariate, we can obtain $\widehat{\operatorname{Risk}}\left(Y_{i}\right), i=1, \ldots, n$ using say pseudomaximum likelihood method, and then estimate the covariate-specific distribution of $\operatorname{Risk}(Y)$ by plugging $\widehat{\operatorname{Risk}}\left(Y_{i}\right)$ into the weighted semiparametric location-scale model for logit $\{\operatorname{Risk}(Y)\}$.

Note that from a matched case-control biomarker study, there are other types of predictiveness curves we might be interested in estimating besides the covariate-specific predictiveness curve investigated here. For example, we might be interested in the predictiveness curve based on both the biomarker and the covariates. Alternatively, we might want to investigate the covariate-adjusted predictiveness curve for the marker, characterized by a weighted summary of the covariate-specific predictiveness curves with weights related to the covariate distribution. These different types of predictiveness curves can be used to answer different scientific questions. Estimation of these predictiveness curves in a matched case-control study can be achieved by combining estimates of the covariate-specific predictiveness $R_{z}(v)$ vs $v$ and some extra information. This is a topic of current research. 


\section{Acknowledgments}

The authors are grateful for support provided by NIH grants GM-54438 and NCI grants CA86368.

\section{References}

Breslow, N. E. (1996). Statistics in epidemiology: the case-control study. JASA 91,14-28.

Breslow, N. E. and Day, N. E. (1993). Statistical Methods in Cancer Research: The Analysis of Case-Control Studies. IARC.

Breslow, N. E. and Cain, K. C. (1988). Logistic regression for two-stage case-control data. Biometrika 75(1), $11-20$.

Breslow, N. E. and Holubkov, R. (1997). Maximum likelihood estimation of logistic regression parameters under two-phase, outcome-dependent sampling. J. R. Statist. Soc. B 59(2), 447-461.

Breslow, N. E. and Holubkov, R. (1997). Weighted likelihood, pseudo-likelihood and maximum likelihood methods for logistic regression analysis of two-stage data. Statistics in Medicine 16, 103-116.

Breslow, N. E. and Zhao, L. P. (1988). Logistic regression for stratified case-control studies. Biometrics 44, 891-899.

Cole, T. J. and Green, P. J. (1992). Smoothing reference centile curves: The LMS method and penalized likelihood. Statistics in Medicine 11(165), 1305-1319.

Fears, T. R. and Brown, C. C. (1986). Logistic regression methods for retrospective case-control studies using complex sampling procedures. Biometrics, 42, 955-960.

Flanders, W. D. and Greenland, S. (1991). Analytic methods for two-stage case-control studies and other stratified designs. Statistics in Medicine, 10, 739-747.

Cook, N. R. (2007). Use and misuse of the receiver operating characteristic curve in risk prediction. Circulation 115, 928-935.

Heagerty, P. J. and Pepe, M. S. (1999). Semiparametric estimation of regression quantiles with application to standardizing weight for height and age in U.S. children. Applied Statistics 48, 553-551. 
Horvitz, D. G. and Thompson, D. J. (1952). A generalization of sampling without replacement from a finite universe. JASA 47, 663-685.

Hsieh, D. A. and Manski, C. F. and McFadden, D. (1985). Estimation of response probabilities from augmented retrospective observations. JASA, 80(391), 651-662.

Huang, Y. and Pepe, M. S. and Feng, Z. (2007). Evaluating the predictiveness of a continuous marker. Biometrics 63(4), 1181-1188.

Huang, Y. (2007). Evaluating the predictiveness of continuous biomarkers. $U W$ thesis.

Janssens, A.C., Deng, Y., Borsboom, G.J., Eijkemans, M.J., Habbema, J.D., and Steyerberg, E.W. (2005). A new logistic regression approach for the evaluation of diagnostic test results. Medical Decision Making, 25, 168-177.

Krijnen, P., van Jaarsveld, B.C., Steyerberg, E.W., Man in 't Veld, A.J., Schalekamp, M.A., and Habbema, J.D. (1998). A clinical prediction rule for renal artery stenosis. Ann Intern Med, 129, 705-711.

Manski, C. F. and McFadden, D. (1991). Alternative Estimators and Sample Designs for Discrete Choice Analysis. Structural Analysis of Discrete Data with Econometric Applications, 2-50.

Owen, A. B. (1988). Empirical likelihood ratio confidence intervals for a single functional. Biometrika 75(2), $237-249$.

Owen, A. B. (1990). Empirical Likelihood Ratio Confidence Regions. The Annals of Statistics 18(1), 90-120.

Pepe, M. S. (2003). The Statistical Evaluation of Medical Tests for Classification and Prediction. Oxford University Press.

Pepe, M. S. and Etzioni, R. and Feng, Z. and Potter, J. D. and Thompson, M. L. and Thornquist, M. and Winget, M. and Yasui, Y. (2001) Phases of biomarker development for early detection of cancer. Journal of the National Cancer Institute 93(14), 1054-1061.

Pepe, M. S. and Feng, Z. and Huang, Y. and Longton, G. M. and Prentice, R. and Thompson, I. M. and Zheng, Y. (2007). Integrating the predictiveness of a marker with its performance as a classifier. American Journal of Epidemiology (In Press). 
Prentice, R. L. and Pyke, R. (1979). Logistic Disease Incidence Models and Case-Control Studies. Biometrika 66(3), 403-411.

Qin, J. and Lawless, J. (1994). Empirical likelihood and general estimating equations. The Annals of Statistics $\mathbf{2 2 ( 1 ) , 3 0 0 - 3 2 5 . ~}$

Scott, A. and Wild, C. (1997). Fitting regression models to case-control data by maximum likelihood. Biometrika 84(1), 57-71.

Scott, A. and Wild, C. (2001). Case-control studies with complex sampling. Biometrika 50, 389-401.

Wild, C. J. (1991). Fitting prospective regression models to case-control data. Biometrika 78(4), 705-717.

\section{Appendix}

\section{A1: Proof of Theorems 1 and 2}

\section{Proof of Theorem 1}

By Taylor's expansion,

$$
\begin{aligned}
\sqrt{n}\left\{\hat{R}_{z}(v)-R_{z}(v)\right\} & =\sqrt{n}\left[G\left\{\hat{\theta}, \hat{F}_{z}^{-1}(v), z\right\}-G\left\{\theta, F_{z}^{-1}(v), z\right\}\right] \\
& =\left\{\left.\frac{\partial G(s, y, z)}{\partial y}\right|_{s=\theta, y=F_{z}^{-1}(v)}\right\}^{T} \sqrt{n}\left\{\hat{F}_{z}^{-1}(v)-F_{z}^{-1}(v)\right\} \\
& +\left(\left.\frac{\partial G(s, y, z)}{\partial s}\right|_{s=\theta, y=F_{z}^{-1}(v)}\right)^{T} \sqrt{n}(\hat{\theta}-\theta)+o_{p}(1) .
\end{aligned}
$$

The result follows according to the delta method. Asymptotic normality of $\hat{\theta}$ and $\hat{F}_{z}$ follows similar arguments as in Huang (2007).

\section{Proof of Theorem 2}

$$
\begin{aligned}
& \sqrt{n}\left\{\hat{R}_{z}^{-1}(p)-R_{z}^{-1}(p)\right\}=\sqrt{n}\left[\hat{F}_{z}\left\{G^{-1}(\hat{\theta}, p, z)\right\}-F_{z}\left\{G^{-1}(\theta, p, z)\right\}\right] \\
= & \sqrt{n}\left[\hat{F}_{z}\left\{G^{-1}(\theta, p, z)\right\}-F_{z}\left\{G^{-1}(\theta, p, z)\right\}\right]+\sqrt{n}\left[F_{z}\left\{G^{-1}(\hat{\theta}, p, z)\right\}-F_{z}\left\{G^{-1}(\theta, p, z)\right\}\right] \\
+ & R_{n},
\end{aligned}
$$


where

$$
\begin{aligned}
R_{n} & =\sqrt{n}\left[\hat{F}_{z}\left\{G^{-1}(\hat{\theta}, p, z)\right\}-\hat{F}_{z}\left\{G^{-1}(\theta, p, z)\right\}\right] \\
& -\left(\sqrt{n}\left[F_{z}\left\{G^{-1}(\hat{\theta}, p, z)\right\}-F_{z}\left\{G^{-1}(\theta, p, z)\right\}\right]\right)=o_{p}(1)
\end{aligned}
$$

by equicontinuity of the process $\sqrt{n}\left(\hat{F}_{z}-F_{z}\right)$. The result follows according to the delta method.

\section{A2: Estimation without including main effect for each covariate group}

Start from (6)

$$
p_{i z}=\frac{1}{n_{z}+\lambda_{2 z}\left\{\mathcal{L} \mathrm{R}_{z}\left(Y_{i}, Z_{i}\right)-1\right\}}=\frac{1}{n_{z}+\lambda_{2 z}\left[\exp \left\{\theta_{0}+\eta(z)+\theta_{1} Y_{i}+\theta_{2}^{T} Z_{i}^{M}+\theta_{3}^{T} Y_{i} Z_{i}^{I}\right\}-1\right]}
$$

and

$$
\sum_{i \in U_{z}} \frac{\exp \left\{\theta_{0}+\eta(z)+\theta_{1} Y_{i}+\theta_{2}^{T} Z_{i}^{M}+\theta_{3}^{T} Y_{i} Z_{i}^{I}\right\}-1}{n_{z}+\lambda_{2 z}\left[\exp \left\{\theta_{0}+\eta(z)+\theta_{1} Y_{i}+\theta_{2}^{T} Z_{i}^{M}+\theta_{3}^{T} Y_{i} Z_{i}^{I}\right\}-1\right]}=0
$$

Substituting $p_{i z}$ into (5) we have

$$
\begin{aligned}
l & =\sum_{z=1}^{\mathcal{Z}} \sum_{i \in U_{z}}-\log \left(n_{z}+\lambda_{2 z}\left[\exp \left\{\theta_{0}+\eta(z)+\theta_{1} Y_{i}+\theta_{2}^{T} Z_{i}^{M}+\theta_{3}^{T} Y_{i} Z_{i}^{I}\right\}-1\right]\right) \\
& +\sum_{z=1}^{\mathcal{Z}} \sum_{j \in U_{D z}}\left\{\theta_{0}+\eta(z)+\theta_{1} Y_{i}+\theta_{2}^{T} Z_{i}^{M}+\theta_{3}^{T} Y_{i} Z_{i}^{I}\right\}
\end{aligned}
$$

Thus the maximum likelihood estimators $\hat{\theta}_{0}$ and $\hat{\theta}_{1}$ solve

$$
\begin{aligned}
\frac{\partial l}{\partial \theta_{0}} & =n_{D}-\sum_{z=1}^{m} \lambda_{2 z} \sum_{i \in U_{z}} p_{i z} \exp \left\{\theta_{0}+\eta(z)+\theta_{1} Y_{i}+\theta_{2}^{T} Z_{i}^{M}+\theta_{3}^{T} Y_{i} Z_{i}^{I}\right\} \\
& =n_{D}-\sum_{z=1}^{\mathcal{Z}} \lambda_{2 z} \sum_{i \in U_{z}} \frac{\exp \left\{\theta_{0}+\eta(z)+\theta_{1} Y_{i}+\theta_{2}^{T} Z_{i}^{M}+\theta_{3}^{T} Y_{i} Z_{i}^{I}\right\}}{n_{z}+\lambda_{2 z}\left[\exp \left\{\theta_{0}+\eta(z)+\theta_{1} Y_{i}+\theta_{2}^{T} Z_{i}^{M}+\theta_{3}^{T} Y_{i} Z_{i}^{I}\right\}-1\right]}=0, \\
\frac{\partial l}{\partial \theta_{1}} & =n_{D}-\sum_{z=1}^{m} \lambda_{2 z} \sum_{i \in U_{k}} p_{i z} Y_{i} \exp \left\{\theta_{0}+\eta(z)+\theta_{1} Y_{i}+\theta_{2}^{T} Z_{i}^{M}+\theta_{3}^{T} Y_{i} Z_{i}^{I}\right\} \\
& =n_{D}-\sum_{z=1}^{\mathcal{Z}} \lambda_{2 z} \sum_{i \in U_{z}} Y_{i} \frac{\exp \left\{\theta_{0}+\eta(z)+\theta_{1} Y_{i}+\theta_{2}^{T} Z_{i}^{M}+\theta_{3}^{T} Y_{i} Z_{i}^{I}\right\}}{n_{z}+\lambda_{2 z}\left[\exp \left\{\theta_{0}+\eta(z)+\theta_{1} Y_{i}+\theta_{2}^{T} Z_{i}^{M}+\theta_{3}^{T} Y_{i} Z_{i}^{I}\right\}-1\right]}=0 .
\end{aligned}
$$

Observe that these are the score equations if we fit a prospective logistic model

$$
\operatorname{logit}\{P(D=1 \mid Y, Z=z)\}=\theta_{0}+\xi(z)+\theta_{1} Y_{i}+\theta_{2}^{T} Z_{i}^{M}+\theta_{3}^{T} Y_{i} Z_{i}^{I}
$$

to the case-control sample using $\xi(z)=\log \left\{\lambda_{2 z} /\left(n_{z}-\lambda_{2 z}\right)\right\}+\eta(z)$ as the offset term. Therefore, one way to estimate $\theta$ is to iteratively estimate $\lambda_{2 z}$ and $\theta$. That is, given some starting value of $\lambda_{2 z}$, we fit the modified 
logistic regression model to obtain an estimate of $\theta$. Given the current estimate of $\theta$, a new $\hat{\lambda}_{2 z}$ is solved as the solution to (11). Continue till convergence. 